\title{
Prediction for the Negative Conversion Probability of Nucleic Acid Testing in Patients with Nonsevere COVID-19 Pneumonia: A Model Based on Retrospective Cohort Study
}

\section{Facai Zhang}

Sichuan University West China Hospital

Huan Hu

Guizhou Medical University

Xiaoming Wang

Sichuan University West China Hospital

Xiaodan Li

Affiliated Hospital of Guiyang Medical University

\section{Yang Yang}

Guizhou Medical University

Li Li

Affiliated Hospital of Guiyang Medical University

Ping Han ( $\square$ hanpingwch@163.com )

Sichuan University West China Hospital https://orcid.org/0000-0002-9710-0037

Long Li

Affiliated Hospital of Guiyang Medical University

\section{Research Article}

Keywords: coronavirus, COVID-19, prediction, nomogram

Posted Date: May 19th, 2020

DOI: https://doi.org/10.21203/rs.3.rs-29277/v1

License: (c) (i) This work is licensed under a Creative Commons Attribution 4.0 International License. Read Full License 


\section{Abstract}

Background: We aimed to screen clinical independent predictive factors for negative conversion of nucleic acid testing and established a predictive nomogram, so as to relieve patients' anxiety and reduce unnecessary repeated nucleic acid testing.

Methods: All 70 consecutive patients with nonsevere COVID-19 pneumonia were admitted to the Fangcang shelter hospital in Wuhan from February $12^{\text {th }}$ to March $8^{\text {th }}, 2020$. We used univariate KaplanMeier analysis and univariate and multivariate Cox regression to identify independent predictive factors and refit the predictive model. Area under ROC (AUR), Brier scores and calibration plots were used to assess the performance.

Results: diabetes mellitus, gender and lymphocyte were deemed independent predictive factors and were incorporated into a Cox proportional hazards model. The AUR and Brier scores of the predictive model at 14 days were $0.694[0.472 ; 0.890]$ and $0.163[0.109 ; 0.219]$ in the internal validation set, respectively. Similarly, the AUR and Brier scores at 21 days were $0.779[0.505 ; 0.957]$ and $0.105[0.042 ; 0.175]$ in the internal validation set.

Conclusions: By using the predictive nomogram, the clinicians could inform patients with nonsevere COVID-19 regarding a certain time to possible negative conversion, which would relieve the patients' anxiety and reduce repeated testing.

\section{Introduction}

In December 2019, an unexplained pneumonia emerged in Wuhan and a new ßcoronavirus was identified by nucleic acid tests, which was named SARS-CoV-2. Currently, COVID-19 is spreading rapidly worldwide and is defined as a pandemic by World Health Organization (WHO) (1). There have been 2.24 million confirmed cases worldwide and more than 150,000 deaths as of April $19^{\text {th }} 2020$ (2). In response to the novel coronavirus outbreak, China first proposed the concept of "Fangcang shelter hospitals" in early February and immediately put it into use to solve the major public health problem, namely, the shortage of isolated hospital wards. The stadium, exhibition center and other public places were transformed into Fangcang shelter hospitals, large temporary hospitals, to isolate nonsevere infectious patients and provide necessary medical services (3).

A wealth of clinical data is urgently needed for a better understanding of the disease. By studying 174 consecutive patients with COVID-19, Guo (4) found that diabetes is an independent risk factor for disease progression or deterioration. Qin (5) compared 286 severe COVID-19 patients and 166 nonsevere COVID19 patients, and found that an increased neutrophil-lymphocyte-ratio and low lymphocyte count were the risk factors that were significant in monitoring the progression of disease. Gender and age were also believed to be independent risk factors for deterioration in some articles (6-8). A CALL model (9) was fitted to predict the risk of patients with stable COVID-19 becoming severe. 
Approximately $80 \%$ of patients with COVID-19 have mild symptoms, such as cough, weakness, nasal obstruction, rhinorrhea and pharyngalgia, and do not need special treatment (10). Additionally patients with nonsevere COVID-19 quarantined from the public often feel anxious and desperate(11). When nucleic acid testings convert to negative outcomes truly matters for patients, because the negative conversion is a significant part of discharge criteria or recovery. Therefore an objective and practical model is necessary to help provide nonsevere patients with a rough estimate and it could relieve patients' anxiety, improve doctor-patient communication, and reduce unnecessary repeated nucleic acid testings. As we known, the model we established is the first to predict the negative conversion of nucleic acid testings.

\section{Methods}

\section{Study Design and Characteristics of Participants}

This retrospective cohort study was approved by the Ethics Committee of the Affiliated Hospital of Guizhou Medical University (AHGMU), Guizhou (2020237). Due to the retrospective nature of the study and emerging infectious disease, written informed consent was waived by the Ethics Committee of AHGMU. We doctors were transferred to Wuhan to support and treat patients with COVID-19 pneumonia in the Fangcang shelter hospital from February $12^{\text {th }}$ to March $8^{\text {th }}$ in 2020 . COVID-19 patients were diagnosed according to Chinese COVID-19 pneumonia guidelines (trial version 5) (12) and the WHO interim guidelines (13). The patients admitted to the Fangcang hospital should meet the criteria (6): (1) positive outcomes of SARS-CoV-2 RT-PCR test; (2) breathing rate $<30$ times per minute and blood oxygen saturation $>93 \%$ on room air; (3) age $\geq 16$ and having self-care ability; and (4) negative outcomes of an influenza virus RT-PCR test. Additionally, COVID-19 patients with the following conditions cannot be admitted to the Fangcang hospital: (1) severe comorbidities, such as acute myocardial infarction, acute cerebral hemorrhage, acute cerebral infarction, heart failure, renal failure and so forth. (2) severe mental and psychological disorder. (3) other severe infectious diseases. Generally, COVID-19 patients in the Fangcang hospital are in concordance with "mild illness patients" or "pneumonia patients" according to the classification of WHO clinical syndromes associated with COVID-19 (13). Nonsevere COVID-19 patients were defined as those admitted to the Fangcang shelter hospital.

\section{Procedure}

All suspected COVID-19 patients were quarantined and underwent nucleic acid testings in local primary care organization. Once RT-PCR confirmed the presence of SARS-CoV-2 in nasopharyngeal swab samples, patients who met the above inclusion and exclusion criteria were transferred to the Fangcang shelter hospital and received antivirus therapy, traditional Chinese medicine treatment (Figure 1).

\section{Data Collection}

The retrospective cohort study started on February $12^{\text {th }}$ and ended on March $8^{\text {th }}$ in 2020. After admission to our Fangcang shelter hospital, we collected 70 consecutive patients' general conditions, symptoms, 
underlying diseases and basic laboratory tests. General conditions included age, gender, temperature and so forth. Symptoms consisted of cough, weakness, nasal obstruction, rhinorrhea, pharyngalgia, chest distress, diarrhea and dyspnea. Underlying diseases included hypertension, stable coronary heart disease (CHD) and diabetes mellitus (DM) for at least 6 months. Basic laboratory tests recorded only the lymphocyte count and WBC count. All factors mentioned above were regarded as predictive variables and were recorded by two clinicians independently. The outcomes variables of our study were the negative conversion time of nucleic acid testing and the negative outcomes of the tests. The negative conversion time of nucleic acid testing refers to the period from the first positive outcomes of nucleic acid testing to negative outcomes of two consecutive nucleic acid tests and the negative outcomes were confirmed by RT-PCR twice. All patients in our cohort were followed up until March $13^{\text {th }}, 2020$.

\section{Statistical Analysis}

Statistical analyses were performed using R software, version 3.6.1 (R Foundation for Statistical Computing, Vienna, Austria). Continuous predictive variables, such as temperature, WBC count, and lymphocyte count, were converted to categorical variables in light of normal values. According to the relationship between predictive variables and outcomes (Supplementary Figure 1), age was converted to a ternary variable: $\leq 40,40-50, \geq 50$ years of age.

All recorded factors mentioned above were screened first by univariate Cox analysis and univariate Kaplan-Meier analysis. The factors with statistical significance $(p<0.05)$ were incorporated into a multivariate analysis and then the independent variables mentioned as risk factors for COVID-19 progression in published articles were also incorporated. Additionally, factors believed to be predictive factors for recovery by specialists were included in the multivariate analysis as well.

The incorporated variables in the multivariate Cox regression model were screened by lasso to avoid overfitting. The remaining variables were deemed the final predictive factors, and the final Cox proportional hazard model was refitted. We made the model diagnosis based on the proportional hazards assumption, influential cases and multicollinearity. The bootstrap strategy was used to validate the model internally. The model was presented with a nomogram to facilitate clinical practice. AUR, Brier scores and calibration plots were used to assess the performance of the model at 14 and 21 days.

\section{Results}

\section{Clinical characteristics of the patients}

70 consecutive patients were admitted to our Fangcang shelter hospital from February $12^{\text {th }}$ to March $8^{\text {th }}$, 2020. 67 of 70 (Approximately 95.7\%) patients had two consecutive negative outcomes of nucleic acid testings and the average negative conversion time was $17.50 \pm 3.87$ days. $32(46 \%)$ patients were male and the median age of patients was 50 [40-57] years. The median WBC count of patients was 5.26 [4.06$6.44]^{\star} 10^{\wedge} 9 / \mathrm{L}$ and the average number of lymphocytes was $1.30 \pm 0.49^{*} 10^{\wedge} 9 / \mathrm{L}$. The number of patients with a normal temperature was $14(20 \%)$, and the others had different levels of fever. The numbers of 
patients with cough, weakness, nasal obstruction, rhinorrhea, pharyngalgia, chest distress, diarrhea, and dyspnea were $38(54 \%), 17(24 \%), 3(4 \%), 4(6 \%), 6(9 \%), 18(26 \%), 11(16 \%)$, and $8(11 \%)$, respectively. There were $12(17 \%), 10(14 \%), 2(3 \%)$ patients with hypertension, diabetes mellitus and CHD, respectively (Table 1). Additionally, the number of patients with negative conversion at 14 days and 21 days was shown in Table 1.

\section{Independent Predictive Factors Associated with Negative Conversion}

16 recorded factors were first screened by univariate Cox analysis and univariate Kaplan-Meier analysis (Table 1, 2). The factors with statistical significance $(p<0.05)$ were age, gender and DM status (Figure 2 ). Lymphocyte count and temperature were mentioned as risk factors for COVID-19 progression or deterioration in recently published articles $(5,9,14)$. Cough and dyspnea were also believed to be predictive factors for recovery in light of specialists' suggestions.

Finally, age (HR 0.92, 95\% Cl 0.66 - 1.29), gender (HR 2.35, 95\% Cl $1.31-4.21)$, DM (HR $0.40,95 \% \mathrm{Cl}$ 0.19 - 0.85), lymphocyte count (HR 2.28, 95\% Cl $1.11-4.70)$, temperature (HR 1.10, 95\% $\mathrm{Cl} 0.82-1.49$ ), cough (HR 1.16, 95\% Cl $0.66-2.03$ ) and dyspnea (HR 1.04, 95\% Cl $0.44-2.47$ ) were incorporated into a multivariate Cox regression model (Table 2) (Figure 3).

7 variables associated with negative conversion of nucleic acids testing in multivariate Cox regression model were screened by lasso again to avoid overfitting (Supplementary Figure 2, 3). The remaining 3 variables (Gender, lymphocyte count and DM) were deemed the final predictive factors and the Cox proportional hazard model was refitted (Table 3).

\section{Predictive Model for the Probability of Negative Conversion}

We made the model diagnosis and found no influence cases (Supplementary Figure 4) or multicollinearity $(\mathrm{VIF}<2)$. All 3 variables met the proportional hazards assumption (Supplementary Figure 5 ). The bootstrap strategy was used to validate the model internally. The predictive model showed a certain level of accuracy in estimating the probability of negative conversion of nucleic acid testing, with a Cindex of $0.664(95 \% \mathrm{Cl} 0.60-0.74)$.

ROC curves were used to assess the discrimination of the predictive model, while Brier scores and calibration plots were used for calibration of the model. The AUR and Brier scores of the predictive model at 14 days were $0.696[0.558 ; 0.833$ ] and 0.152 [0.099; 0.205], respectively, in the training set (Supplementary Figure 6), while the values were $0.694[0.472 ; 0.890]$ and $0.163[0.109 ; 0.219]$ in the internal validation set after 100 bootstraps (Figure 4). Similarly, the AUR and Brier scores at 21 days were $0.785[0.640 ; 0.930]$ and 0.097 [0.050; 0.144], respectively, in training set (Supplementary Figure 6), and $0.779[0.505 ; 0.957]$ and $0.105[0.042 ; 0.175]$ in the internal validation set after 100 bootstraps (Figure 4 ).

Finally, the model was presented with a nomogram to facilitate clinical practice (Figure 4). Each variable in the nomogram is assigned to a certain score on a point scale from 0 to 100 according to its weight in the model. Patients with nonsevere COVID-19 pneumonia could add the points of each variable and 
calculate the total scores. Then the total scores were projected to the risk lines of 14-day or 21-day positive probability and the negative conversion probability equaled to one minus positive probability.

\section{Discussion}

The Fangcang shelter hospital, a new concept applied by China to cope with serious public health problems, is used to accommodate nonsevere COVID-19 pneumonia patients during the SARS-CoV-2 outbreak(3). The exponentially increasing number of patients with COVID-19 aggravated the burden of global health care resources. Age, gender, temperature, comorbidities and some laboratory tests were deemed independent risk factors for disease progression (4-8), and there have been 10 prognostic models for predicting the severe progression risk or mortality risk before March $24^{\text {th }}, 2020$ (8). Moreover, the majority of patients has mild symptoms and does not need special medical intervention (10). Hence a model that could predict the probability of negative conversion of nucleic acid testing for mild patients becomes important, because it is a significant part of recovery or discharge standards and could relieve the patient's anxiety, reduce repeated valueless testings, and decrease waste of public health resources.

We collected 70 patients' general clinical characteristics and routine blood examinations results in the Fangcang shelter hospital. All factors were screened by univariate analysis and the factors that were statistically significant $(P<0.05)$ or verified as predictors for progression by other articles were incorporated into a multivariate Cox regression. Gender, DM and lymphocyte count were deemed independent predictors and used to refit the final model. In our predictive model, diabetes and low lymphocyte count were independent risk factors for reducing the probability of negative conversion of testings, which were in concordance with factors related to disease progression(4-6). It is speculated that dysregulation of the immune response plays an important role in the pathogenesis of COVID 19 and the lymphocyte count decrease in blood may be caused by the transfer of lymphocytes in peripheral blood to the lungs (15).

Dysregulation of the immune system in diabetic patients may be susceptible to infection(16) and be related to the prolonged nucleic acid detection. Regarding the mechanism, the inhibition of neutrophil chemotaxis, altered cytokine, phagocytic cell dysfunction and hyperglycemia may contributed the immune dysfunction (17). Compared with those without diabetes, diabetic patients were more likely to progress to severe illness and had a higher fatality rate $(4,18)$ and the majority of retrospective cohort studies verified that diabetes was a risk factor for disease progression $(4,19)$.

Interestingly, Ji (9) and Wang (6) believed that being female was a protective factor against disease progression, which in our models was deemed a disadvantage factor for negative conversion. Regarding the reasons, We think it is based on the different composition ratio of patients. The patients in our Fangcang hospital were not only confirmed by RT-PCR, but also met the inclusion and exclusion critiria. In Wang's study, gender in different groups was compared by only univariate analysis ( $c^{2}$ test), so the influence of other confounding factors cannot be ruled out without multivariate analysis. 
Our predictive model had relatively good performance on the 14-day and 21-day internal validation sets. The AUR and Brier score were $0.694[0.472 ; 0.890]$ and $0.163[0.109 ; 0.219]$ in internal validation after 100 bootstrap samplings, which were $0.779[0.505 ; 0.957]$ and $0.105[0.042 ; 0.175]$ at 21 days, respectively.

There are several limitations in our study. First, the concordance index cannot reach very high level, and the probability of negative conversion in low-risks groups of patients is underestimated at 21 days. Second, the sample size is small, so whether the results can be applicable to patients in Hubei Province or other countries still need external valibration. Finally, additional independent predictors incorporated into our model might improve the model's performance, but also restrict its application.

\section{Conclusions}

When using the objective and practical model nomogram, the clinicians could inform patients with nonsevere COVID-19 regarding a certain time to possible negative conversion, which would relieve the patients' anxiety, improve doctor-patient communication, and reduce unnecessary repeated nucleic acid testing. To a certain extent, these saved detection kits could be used to diagnose more suspected patients.

\section{Declarations}

\section{Ethics approval and consent to participate}

This retrospective cohort study was approved by the Ethics Committee of the Affiliated Hospital of Guizhou Medical University (AHGMU), Guizhou (2020237). Due to the retrospective nature of the study and emerging infectious disease, written informed consent was waived by the Ethics Committee of AHGMU.

\section{Consent for publication}

Not applicable

\section{Availability of data and materials}

The dataset supporting the conclusions of this article is included as an additional file

\section{Competing interests}

The authors declare that they have no competing interests

\section{Funding}

the 1.3.5 project for disciplines of excellence, West China Hospital, Sichuan University (ZY2016104) and Pillar Program from Department of Science and Technology of Sichuan Province (2018SZ0219) 


\section{Acknowledgments}

We thanks all patients included in our study.

\section{Authors' Contributions}

ZF and $\mathrm{HH}$ designed this study. $\mathrm{HH}$ and $\mathrm{LX}$ collected the epidemiological and clinical data. HH, WX, LX, YY, LL ( $\mathrm{Li} \mathrm{Li}$ ), HP and LL (Long Li) took part in the data collection. ZF drafted the manuscript, and HP revised the final manuscript.

\section{References}

1. World_Health_Organization. WHO audio emergencies coronavirus press conference full and final 11 mar 2020. https://wwwwhoint/docs/default-source/coronaviruse/transcripts/who-audioemergencies-coronavirus-press-conference-full-and-final-11 mar2020pdf?sfvrsn=cb432bb3_2 2020.

2. World_Health_Organization. Coronavirus disease 2019 (COVID-19) Situation Report - 90. https://wwwwhoint/docs/default-source/coronaviruse/situation-reports/20200419-sitrep-90-covid19pdf?sfvrsn=551d47fd_2. 2020.

3. Chen S, Zhang Z, Yang J, Wang J, Zhai X, Bärnighausen T, et al. Fangcang shelter hospitals: a novel concept for responding to public health emergencies. The Lancet. 2020;395(10232):1305-14.

4. Guo W, Li M, Dong Y, Zhou H, Zhang Z, Tian C, et al. Diabetes is a risk factor for the progression and prognosis of COVID-19. Diabetes Metab Res Rev. 2020:e3319.

5. Guo T, Fan Y, Chen M, Wu X, Zhang L, He T, et al. Cardiovascular Implications of Fatal Outcomes of Patients With Coronavirus Disease 2019 (COVID-19). JAMA Cardiology. 2020.

6. Wang X, Fang J, Zhu Y, Chen L, Ding F, Zhou R, et al. Clinical characteristics of non-critically ill patients with novel coronavirus infection (COVID-19) in a Fangcang Hospital. Clinical Microbiology and Infection. 2020.

7. Liu R, Han H, Liu F, Lv Z, Wu K, Liu Y, et al. Positive rate of RT-PCR detection of SARS-CoV-2 infection in 4880 cases from one hospital in Wuhan, China, from Jan to Feb 2020. Clin Chim Acta. 2020;505:172-5.

8. Wynants L, Van Calster B, Bonten MMJ, Collins GS, Debray TPA, De Vos M, et al. Prediction models for diagnosis and prognosis of covid-19 infection: systematic review and critical appraisal. Bmj. 2020.

9. Ji D, Zhang D, Xu J, Chen Z, Yang T, Zhao P, et al. Prediction for Progression Risk in Patients with COVID-19 Pneumonia: the CALL Score. Clin Infect Dis. 2020.

10. World_Health_Organization. Q\&A on coronaviruses (COVID-19): What are the symptoms of COVID19 ? 2020.

11. KH S, M A, A J, Danubina AAJP. COVID-19 Pandemic and Impending Global Mental Health Implications. 2020;32(1):32-5. 
12. Lin L, Li TS. [Interpretation of "Guidelines for the Diagnosis and Treatment of Novel Coronavirus (2019-nCoV) Infection by the National Health Commission (Trial Version 5)"]. Zhonghua yi xue za zhi. 2020;100(0):E001.

13. World_Health_Organization. Clinical management of severe acute respiratory infection when novel coronavirus (nCoV) infection is suspected: interim guidance. 2020.

14. Mo P, Xing Y, Xiao Y, Deng L, Zhao Q, Wang H, et al. Clinical characteristics of refractory COVID-19 pneumonia in Wuhan, China. Clin Infect Dis. 2020.

15. Xu Z, Shi L, Wang Y, Zhang J, Huang L, Zhang C, et al. Pathological findings of COVID-19 associated with acute respiratory distress syndrome. The Lancet Respiratory Medicine. 2020;8(4):420-2.

16. Joshi N, Caputo GM, Weitekamp MR, Karchmer AW. Infections in patients with diabetes mellitus. N Engl J Med. 1999;341(25):1906-12.

17. AM A, MJ B, clinical MCJM, experimental. COVID-19 and diabetes mellitus: what we know, how our patients should be treated now, and what should happen next. 2020:154245.

18. Wu Z, McGoogan JM. Characteristics of and Important Lessons From the Coronavirus Disease 2019 (COVID-19) Outbreak in China: Summary of a Report of 72314 Cases From the Chinese Center for Disease Control and Prevention. Jama. 2020.

19. Zhou F, Yu T, Du R, Fan G, Liu Y, Liu Z, et al. Clinical course and risk factors for mortality of adult inpatients with COVID-19 in Wuhan, China: a retrospective cohort study. The Lancet. 2020;395(10229):1054-62.

\section{Tables}

Table 1. Baseline Clinical Characteristics of COVID-19 Patients and Univariate Kaplan-Meier Analysis. 
Factors

Count (\%) Negative conversion count (\%) P-value

\begin{tabular}{|c|c|c|c|c|}
\hline & & 14days & 21 days & \\
\hline Overall & 70 & $15(21.4)$ & $61(87.1)$ & \\
\hline Gender & & & & 0.039 \\
\hline Female & $38(54)$ & $6(15.8)$ & $31(81.6)$ & \\
\hline Male & $32(460$ & $9(28.1)$ & $30(93.8)$ & \\
\hline Age & & & & $\varangle 0.0001$ \\
\hline$\leq 40$ & 18(26) & $8(44.4)$ & $18(100)$ & \\
\hline $40 \sim 50$ & $19(27)$ & $2(10.5)$ & $11(57.9)$ & \\
\hline$\nabla 50$ & $33(47)$ & $5(15.2)$ & $32(97.0)$ & \\
\hline WBC & & & & 0.98 \\
\hline$\nabla 4^{\prime} 10^{\wedge} 9 / \mathrm{L}$ & $16(23)$ & $3(18.8)$ & 13(81.3) & \\
\hline$\geq 4^{\prime} 10^{\wedge} 9 / \mathrm{L}$ & $54(77)$ & $12(22.2)$ & 48(88.9) & \\
\hline Lymphocyte & & & & 0.1 \\
\hline$\otimes 0.8^{\prime} 10^{\wedge} 9 / \mathrm{L}$ & $14(20)$ & $1(7.1)$ & 11(78.6) & \\
\hline$\geq 0.8^{\prime} 10^{\wedge} 9 / \mathrm{L}$ & $56(80)$ & $14(25.0)$ & $50(89.3)$ & \\
\hline Temperature $\left({ }^{\circ} \mathrm{C}\right)$ & & & & 0.76 \\
\hline$<37.3$ & $14(20)$ & $3(21.4)$ & 11(78.6) & \\
\hline $37.3 \sim 38$ & $25(36)$ & $4(16.0)$ & $23(92.0)$ & \\
\hline $38.1 \sim 39$ & $25(36)$ & $7(28.0)$ & $22(88.0)$ & \\
\hline \39 & $6(8)$ & $1(16.7)$ & $5(83.3)$ & \\
\hline Cough & & & & 0.28 \\
\hline without & $32(46)$ & $4(12.5)$ & $31(96.9)$ & \\
\hline with & $38(54)$ & $11(28.9)$ & $30(78.9)$ & \\
\hline Weakness & & & & 0.26 \\
\hline without & $53(76)$ & $9(17.0)$ & $46(86.8)$ & \\
\hline with & $17(24)$ & $6(35.3)$ & 15(88.2) & \\
\hline Nasal obstruction & & & & 0.2 \\
\hline
\end{tabular}




\begin{tabular}{|c|c|c|c|c|}
\hline without & $67(96)$ & 14(20.9) & $58(86.6)$ & \\
\hline with & $3(4)$ & $1(33.3)$ & $3(100)$ & \\
\hline Rhinorrhoea & & & & 0.3 \\
\hline without & $66(94)$ & $14(21.2)$ & $57(86.4)$ & \\
\hline with & $4(6)$ & $1(25.0)$ & $4(100)$ & \\
\hline Pharyngalgia & & & & 0.91 \\
\hline without & $64(91)$ & 13(20.3) & $56(87.5)$ & \\
\hline with & $6(9)$ & $2(33.3)$ & $5(83.3)$ & \\
\hline Chest distress & & & & 0.64 \\
\hline without & $52(74)$ & $12(23.1)$ & $44(84.6)$ & \\
\hline with & 18(26) & $3(16.7)$ & $17(94.4)$ & \\
\hline Diarrhea & & & & 0.33 \\
\hline without & $59(84)$ & 11(18.6) & $51(86.4)$ & \\
\hline with & 11(16》 & $4(36.4)$ & 10(90.9) & \\
\hline Dyspnea & & & & 0.44 \\
\hline without & $62(89)$ & $13(21.0)$ & $55(88.7)$ & \\
\hline with & $8(11)$ & $2(25.0)$ & $6(75.0)$ & \\
\hline \multicolumn{5}{|l|}{ Complication } \\
\hline Hypertension & & & & 0.73 \\
\hline without & $58(83)$ & $12(20.7)$ & $50(86.2)$ & \\
\hline with & 12(17) & $3(25.0)$ & 11(91.7) & \\
\hline Diabetes Mellitus & & & & 0.0074 \\
\hline without & $60(86)$ & $15(25.0)$ & $55(91.7)$ & \\
\hline with & $10(14)$ & $0(0)$ & $6(60.0)$ & \\
\hline CHD & & & & 0.51 \\
\hline without & 68(97) & $15(22.1)$ & $59(86.8)$ & \\
\hline with & $2(3)$ & $0(0)$ & $2(100)$ & \\
\hline
\end{tabular}

Abbreviations: CHD, coronary heart disease; 
Table 2 Univariate and Multivariate Cox Analysis of Negative Conversion

\begin{tabular}{|c|c|c|c|c|c|c|c|c|}
\hline \multirow[t]{2}{*}{ Parameters } & \multicolumn{4}{|c|}{ Univariate analysis } & \multicolumn{4}{|c|}{ Multivariate analysis } \\
\hline & $\begin{array}{l}\text { Hazard } \\
\text { Ratio }\end{array}$ & $\begin{array}{l}\text { Lower } \\
95 \% \mathrm{Cl}\end{array}$ & $\begin{array}{l}\text { upper } \\
95 \% \mathrm{Cl}\end{array}$ & $P$-Value & $\begin{array}{l}\text { Hazard } \\
\text { Ratio }\end{array}$ & $\begin{array}{l}\text { Lower } \\
95 \% \mathrm{Cl}\end{array}$ & $\begin{array}{l}\text { Upper } \\
95 \% \mathrm{Cl}\end{array}$ & $P$-Value \\
\hline Gender & 1.6 & 1 & 2.7 & 0.046 & 2.35 & 1.31 & 4.21 & 0.004 \\
\hline Age & 0.83 & 0.59 & 1.2 & 0.29 & 0.92 & 0.66 & 1.29 & 0.635 \\
\hline WBC & 1 & 0.56 & 1.8 & 1 & - & - & - & - \\
\hline Lymphocyte & 1.7 & 0.91 & 3.1 & 0.097 & 2.28 & 1.11 & 4.7 & 0.025 \\
\hline Temperature & 1 & 0.78 & 1.3 & 0.91 & 1.1 & 0.82 & 1.49 & 0.517 \\
\hline Cough & 0.74 & 0.45 & 1.2 & 0.23 & 1.16 & 0.66 & 2.03 & 0.602 \\
\hline Weakness & 1.3 & 0.76 & 2.4 & 0.31 & - & - & - & - \\
\hline $\begin{array}{l}\text { Nasal } \\
\text { Obstruction }\end{array}$ & 2.1 & 0.67 & 6.9 & 0.2 & - & - & - & - \\
\hline Rhinorrhoea & 1.8 & 0.64 & 5 & 0.26 & - & - & - & - \\
\hline Pharyngalgia & 1.1 & 0.42 & 2.7 & 0.92 & - & - & - & - \\
\hline $\begin{array}{l}\text { Chest } \\
\text { distress }\end{array}$ & 1.2 & 0.68 & 2.1 & 0.56 & - & - & - & - \\
\hline Diarrhea & 1.3 & 0.7 & 2.6 & 0.38 & - & - & - & - \\
\hline Dyspnea & 0.72 & 0.33 & 1.6 & 0.42 & 1.04 & 0.44 & 2.47 & 0.933 \\
\hline Hypertension & 1.1 & 0.59 & 2.1 & 0.75 & - & - & - & - \\
\hline $\mathrm{CHD}$ & 0.66 & 0.16 & 2.7 & 0.56 & - & - & - & - \\
\hline DM & 0.4 & 0.19 & 0.82 & 0.012 & 0.4 & 0.19 & 0.85 & 0.017 \\
\hline
\end{tabular}

Abbreviations: CHD, coronary heart disease; DM, diabetes mellitus.

Table 3 The Refitted Model with 3 Independent Predictors 


\begin{tabular}{lllll} 
Parameters & \multicolumn{4}{l}{ Multivariate analysis } \\
\cline { 2 - 5 } & Coefficient & Hazard Ratio & $95 \% \mathrm{Cl}$ & P-Value \\
\hline Gender & 0.80 & 2.22 & $0.27-2.95$ & 0.004 \\
\hline Lymphocyte & 0.74 & 2.09 & $0.34-2.18$ & 0.251 \\
\hline DM & -0.91 & 0.40 & $0.37-2.42$ & 0.017
\end{tabular}

Abbreviations: DM, diabetes mellitus

Figures 


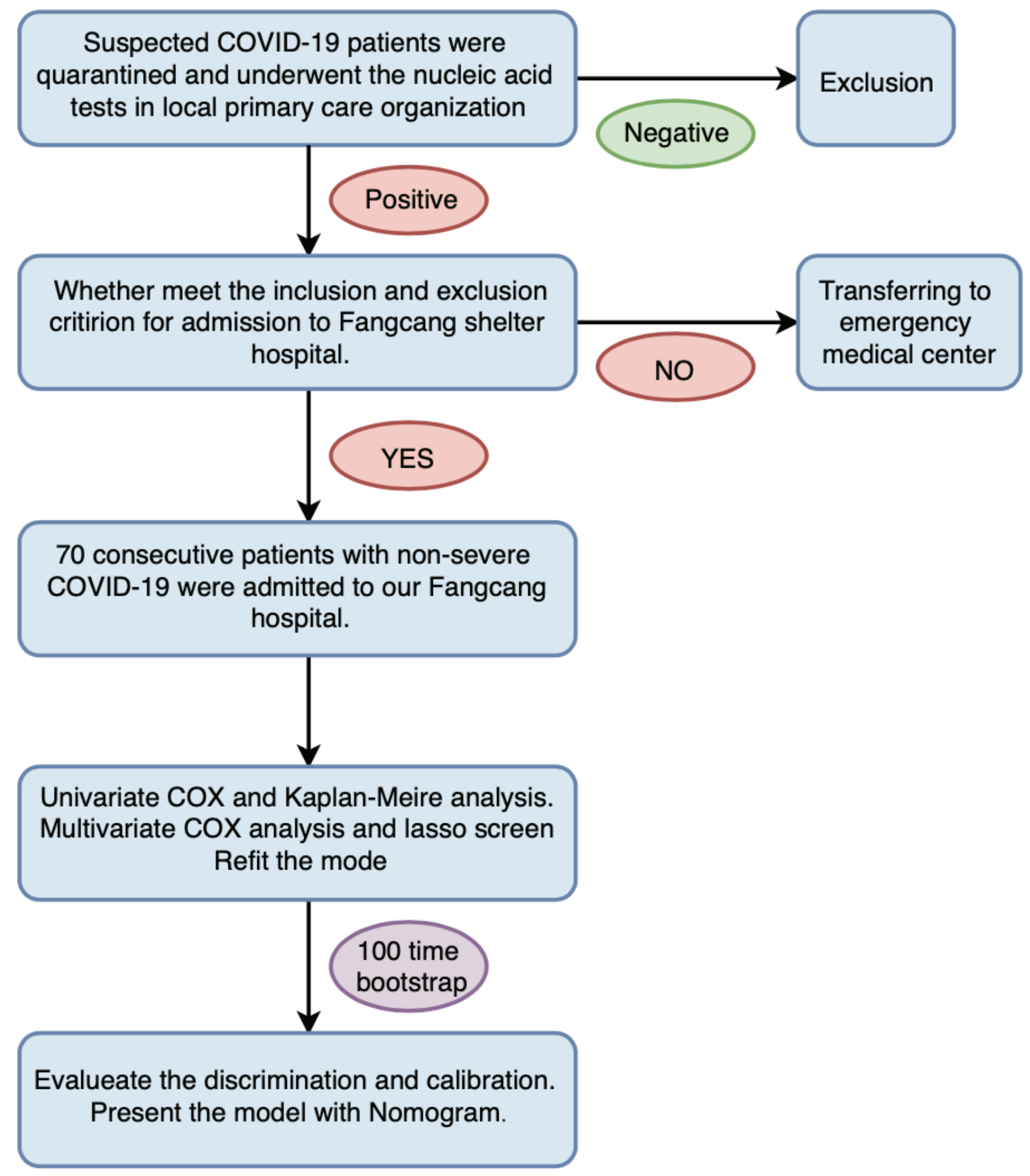

Figure 1

Flow chart for management of patients with COVID-19 

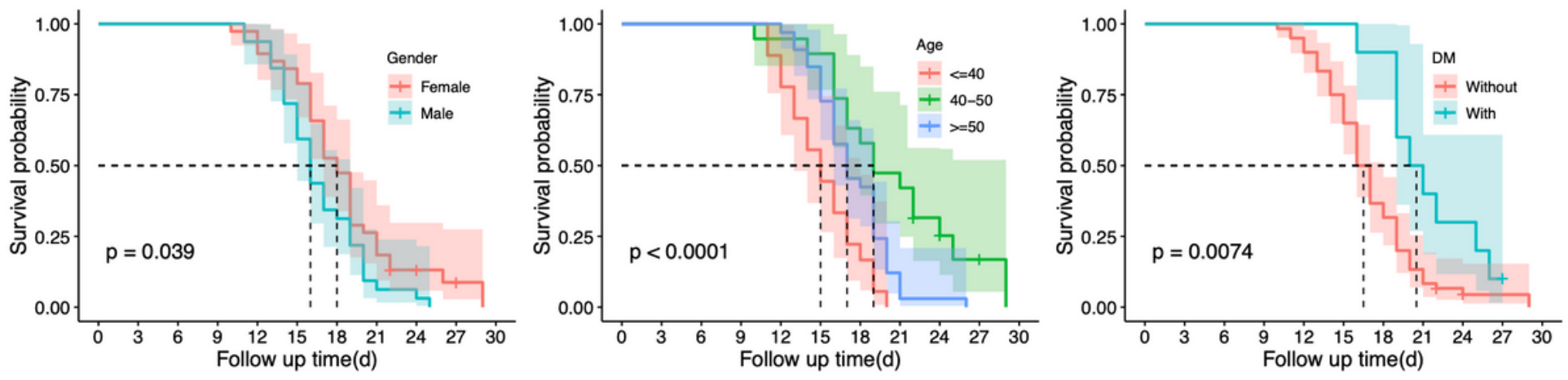

\section{Figure 2}

Univariate Kaplan-Meier Analysis of Negative Conversion. Kaplan-Meier curve shows that age, gender, diabetes mellitus have statistical significance $(P<0.05)$.

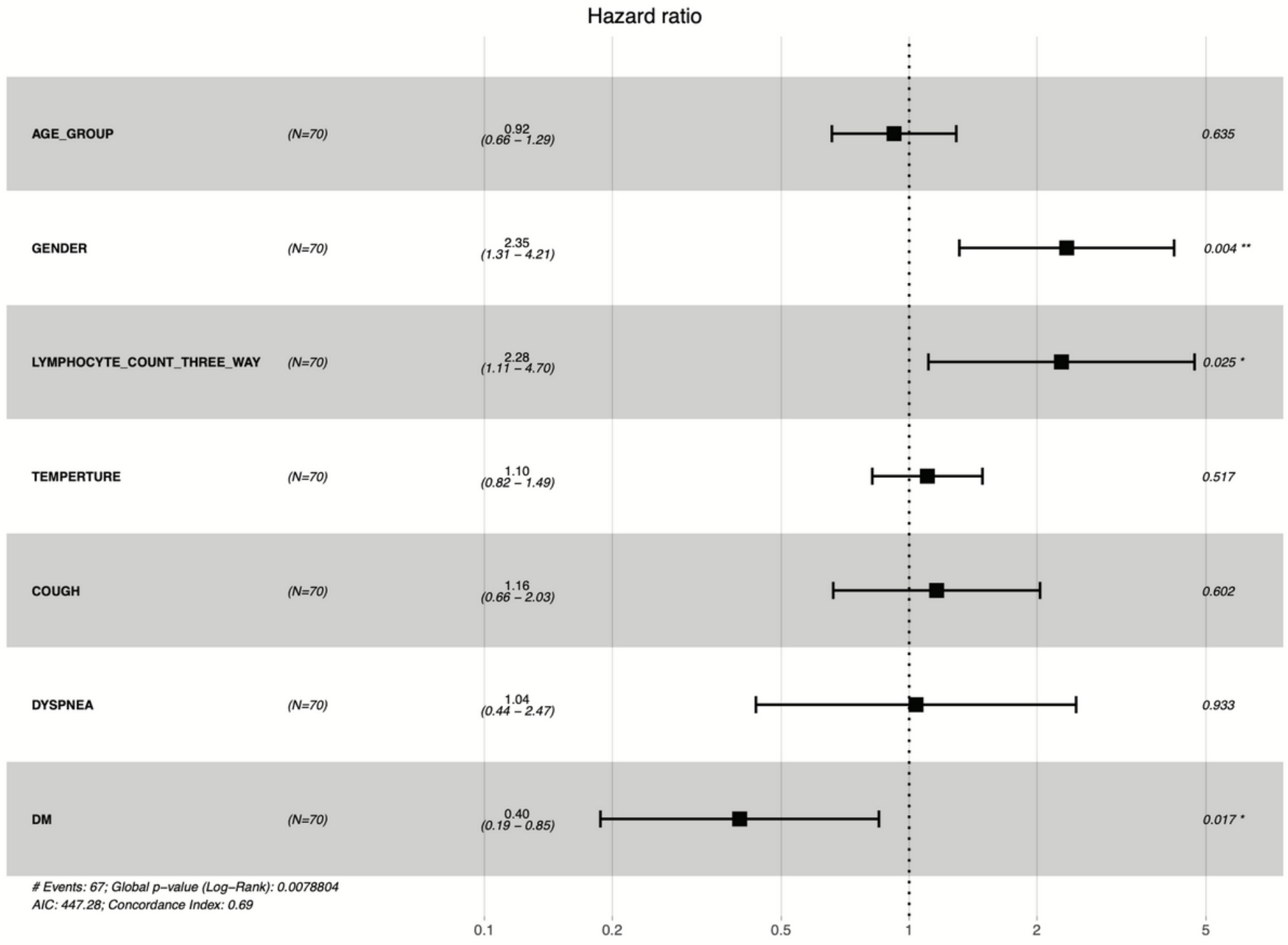

\section{Figure 3}

Hazard Ratio of Multivariate Analysis. 
A

Points

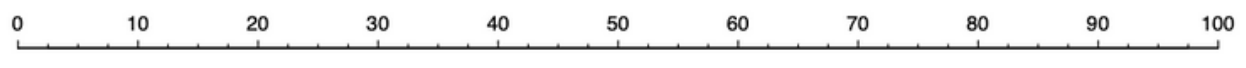

GENDER

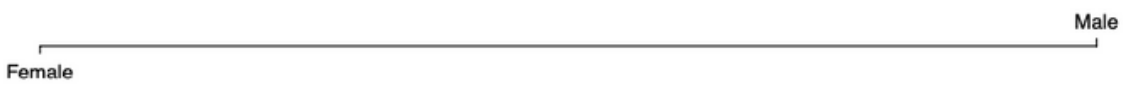

LYMPHOCYTE

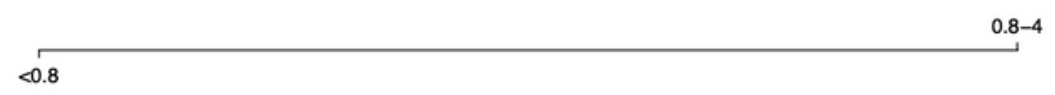

DM

WITHOUT

WITH

Total Points

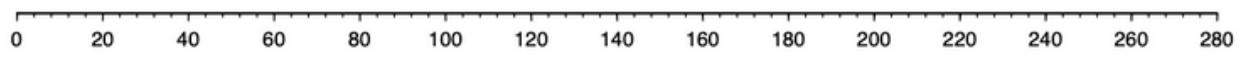

14_Positive

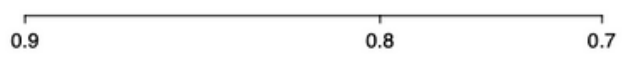

21_Positive

\begin{tabular}{llllll}
\hline 0.6 & 1 & 1 & 1 & 1 & 0.2 \\
\end{tabular}
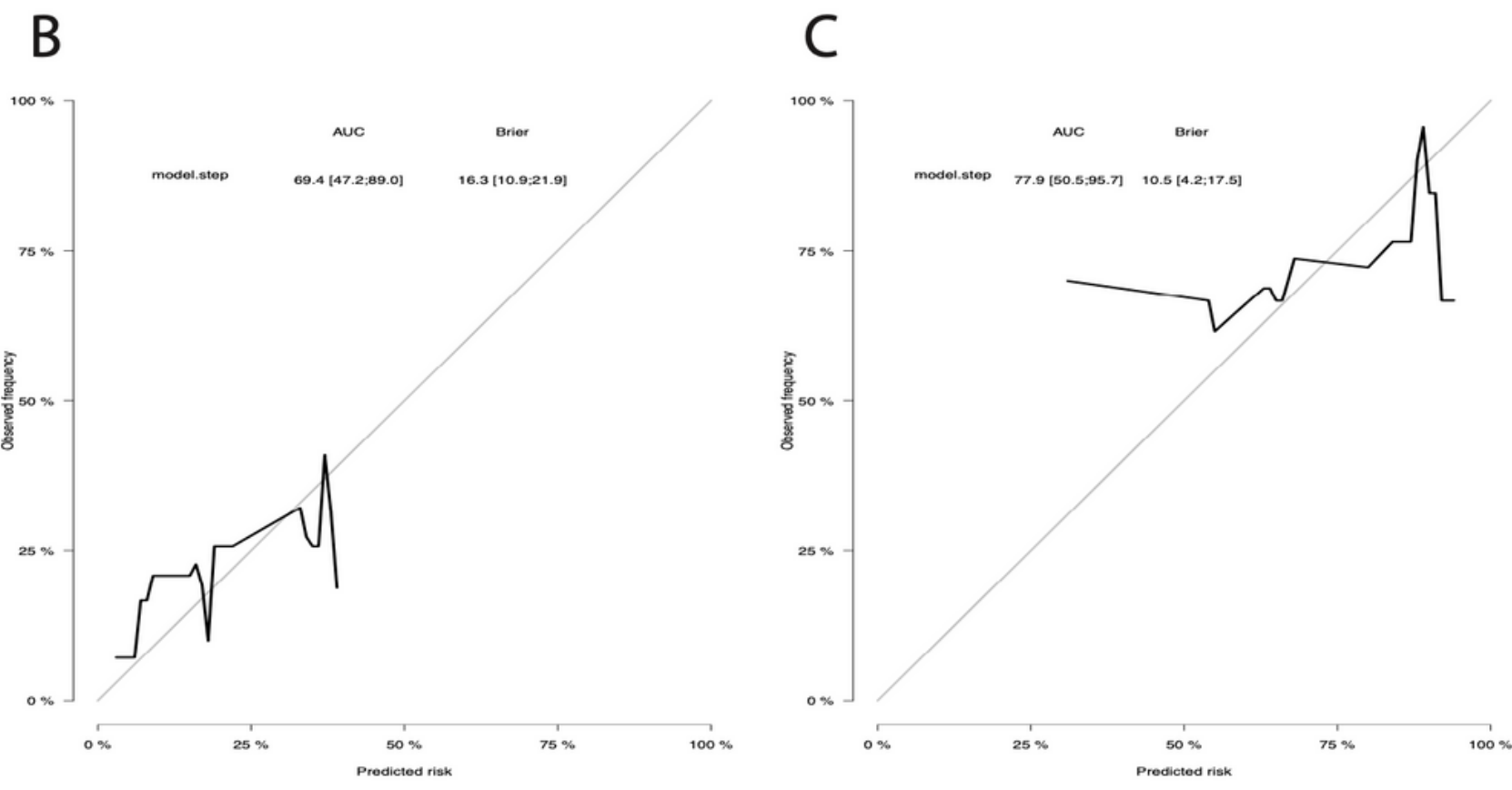

\section{Figure 4}

The Nomogram for Prediction the Probability of Negative Conversion. A. Nomogram to estimate the probability of negative conversion in patients with COVID-19. B. Calibration plot of positive prediction on 14-day in internal validation set after 100 times bootstrap sampling. C. Calibration plot of positive prediction on 21-day in internal-validation set after 100 times bootstrap sampling. The probability of negative conversion in low risks patients is underestimated on 21-day 


\section{Supplementary Files}

This is a list of supplementary files associated with this preprint. Click to download.

- supplementaryfigure6.pdf

- supplementaryfigure4.pdf

- supplementaryfigure3.pdf

- supplementaryfigure2.pdf

- supplementaryfigure1.pdf

- supplementaryfigurelegend.docx

- supplementaryfigure5.pdf 